\title{
Modeling of pulsed-laser guide stars for the Thirty Meter Telescope project
}

\author{
Simon M. Rochester, ${ }^{1, *}$ Angel Otarola, ${ }^{2}$ Corinne Boyer, ${ }^{2}$ Dmitry Budker, ${ }^{3,1}$ Brent Ellerbroek, ${ }^{2}$ \\ Ronald Holzlöhner, ${ }^{4}$ and Lianqi Wang ${ }^{2}$ \\ ${ }^{1}$ Rochester Scientific, LLC, 2041 Tapscott Ave., El Cerrito, California 94530, USA \\ ${ }^{2}$ TMT Observatory Corporation, 1200 East California Boulevard, Mail Code 102-8, Pasadena, California 91125, USA \\ ${ }^{3}$ Department of Physics, University of California, Berkeley, 366 LeConte Hall MC 7300 Berkeley, \\ California 94720-7300, USA \\ ${ }^{4}$ European Southern Observatory (ESO), Garching bei München, D-85748, Germany \\ ${ }^{*}$ Corresponding author: simon@rochesterscientific.com
}

Received April 10, 2012; accepted June 4, 2012;

posted June 20, 2012 (Doc. ID 166420); published August 1, 2012

\begin{abstract}
The Thirty Meter Telescope (TMT) has been designed to include an adaptive optics system and associated laser guide star (LGS) facility to correct for the image distortion due to Earth's atmospheric turbulence and achieve diffraction-limited imaging. We have calculated the response of mesospheric sodium atoms to a pulsed laser that has been proposed for use in the LGS facility, including modeling of the atomic physics, the light-atom interactions, and the effect of the geomagnetic field and atomic collisions. This particular pulsed-laser format is shown to provide comparable photon return to a continuous-wave (cw) laser of the same average power; both the cw and pulsed lasers have the potential to satisfy the TMT design requirements for photon return flux. @ 2012 Optical Society of America
\end{abstract}

OCIS codes: $\quad 350.1260,300.2530,010.1080,010.1310,010.1350$.

\section{INTRODUCTION AND MOTIVATION}

The Giant Segmented Mirror Telescope (GSMT) initiative has been ranked among the highest priorities for U.S. astronomy in the last two decadal surveys of astronomy and astrophysics $[1,2]$. This ranking is motivated by the potential scientific impact of the sensitivity and diffraction-limited resolution that it is possible to achieve with a large-aperture telescope. The Thirty Meter Telescope (TMT) project has designed and is planning the construction of a diffraction-limited, large-aperture segmented telescope on the northern slope of the Mauna Kea volcano in Hawaii (characteristics of the site are summarized in [3]). For such a telescope, adaptive optics (AO) and an associated laser guide star (LGS) system are needed to achieve diffraction-limited capacity.

AO systems are of increasing importance for current [4-ㅛ and future [9-11] ground-based astronomical telescopes. Large ground-based telescopes require $\mathrm{AO}$ to correct for the image distortion induced by atmospheric turbulence. The use of $\mathrm{AO}$ requires a reference light source, such as a bright natural star, in order to measure the effect of the atmosphere on the light wavefront. Often there is no bright natural star available within the isoplanatic angle from the astronomical target, i.e., the angle out to which phase variations in the wavefront are less than $1 \mathrm{rad}$. In this case, a laser can be used to generate an artificial guide star. This can be done by exploiting Rayleighbackscattered light from a pulsed laser [12], or in the case considered here, resonance fluorescence from sodium atoms in the mesosphere $[13,14]$ at 80 to $125 \mathrm{~km}$ altitude, excited by either continuous-wave (cw) or pulsed lasers resonant with the sodium $\mathrm{D}_{2}$ transition at $589 \mathrm{~nm}$. Such techniques were first proposed for astronomy in the 1980s [15,16]; almost 30 years later, LGS systems are available in all 6-10 m-class telescopes.
Many of the key scientific questions to be addressed with TMT [17] - the nature and composition of the universe, the formation of first stars and proto-galaxies, the evolution of galaxies and the interplanetary medium, the relation between black holes and their host galaxies, the formation of stars and planets, the nature of extrasolar planets, and the detection of extrasolar planets within the habitable zone of their host stars-require diffraction-limited imaging and thus an LGSAO system. An example of the need for diffraction-limited imaging is observations of the crowded star field close to our galactic center. Such observations are technically challenging, and astronomers have devised observation strategies to resolve the stellar dynamics and accurately locate and weigh the supermassive black hole in the center of our galaxy. Accurate determinations of the stellar dynamics have evolved significantly since the first survey of this star field by means of speckle imaging in the late 1990s [18], followed by Natural Guide Star-AO in the early 2000s [19]; the best results have been achieved with the use of LGS-AO in the mid- to late 2000s [20]. Yet, there remains the need to improve these observations to resolve stars at angular distances closer to the galactic center. This will allow testing the predictions of general relativity under the gravitational field of the supermassive black hole hosted in the center of our galaxy. This will be made possible by a large-aperture, LGS-AO-assisted telescope, such as TMT.

The TMT LGS facility (LGSF) will initially include six lasers to form an asterism consisting of one LGS on-axis and five LGS equally spaced on a circle of 35 arcsec angular diameter, with the laser-launch telescope (LLT) located behind the secondary mirror [21]. Using the multiconjugate adaptive-optics (MCAO) approach, the LGSF will be able to provide diffraction-limited imaging in a field-of-view from tens of arcseconds 
to about 1-2 arcmin [22]. The LGSF design supports expansion to up to nine lasers to form asterisms for other AO modes and radii from 5 to 510 arcsec [23].

The original design for the TMT LGSF called for cw lasers (either solid-state Nd:YAG sum-frequency lasers or Raman fiber lasers). Subsequently, TMT has also included the option of using pulsed lasers based on diode-pumped solid-state sumfrequency Nd:YAG technology. Such lasers have been developed by scientists at the Technical Institute of Physics and Chemistry of the Chinese Academy of Sciences (TIPC). In this article, we investigate the effectiveness of pulsed lasers for LGS, focusing on the TIPC laser format. We also compare the photon return from the pulsed laser to that of a cw laser of the same average power.

The effectiveness of an LGS system in correcting the turbulence-induced aberrations in the image wavefront depends on having sufficient photon return from the laser beacons. The main requirements set for the first-light TMT AO system are the following: (1) a root-mean-squared (rms) wavefront error at zenith smaller than $187 \mathrm{~nm}$ on-axis and $191 \mathrm{~nm}$ over a 17 arcsec field-of-view, (2) 50\% sky coverage at the galactic pole, (3) operation up to a zenith angle of $65^{\circ}$, and (4) operation under atmospheric turbulence conditions characterized by an atmospheric length scale (Fried length $r_{0}$ [24]) as low as $0.1 \mathrm{~m}$ in the direction of observation. The TMT wavefront reconstruction algorithm [25] has been tested under various scenarios and is expected to meet the maximum wavefront error specifications (see, for instance, [21]), with a significant margin for error, when using a LGS that provides 900 photodetected electrons (PDE) per wavefront sensor subaperture in an integration time of $1.25 \mathrm{~ms}$. The specification of 900 PDE applies when the laser is pointed in the zenith direction, and includes a rough factor of 2 safety margin to account for various effects that have the potential to increase the rms wavefront error, including the effect of directing the laser at larger zenith angles. The photon return scales due to purely geometrical considerations as the cosine of the zenith angle (i.e., the inverse of the airmass); this scaling is used as a reference for comparison with the actual calculated directional dependence of photon return reported here. Some additional factors that affect the actual PDE count are the laser format (power, polarization, spectral characteristics, etc.), various efficiencies in the propagation of the laser light to the launch telescope, atmospheric absorption, the actual column density of sodium atoms in the mesosphere, and the relative direction of the local geomagnetic field with respect to the laser beam. Also important is the efficiency of the LGS detection system (i.e., the wavefront sensor).

Optimizing a laser system to maximize the guide star photon return has been an area of ongoing research for many years. Reference [26] investigated the physical aspects affecting the performance (i.e., photon flux return) of cw sodium lasers. The calculation included the important physical effects of Larmor precession of the sodium atoms in the geomagnetic field, radiation pressure (recoil), and saturation of the optical transitions, as well as velocity-changing and spin-randomizing collisions. This was achieved using a density-matrix calculation with coupled velocity groups. A discussion was given of various mechanisms that are detrimental to photon return; i.e., Larmor precession reduces the efficiency of optical pumping, recoil depopulates the velocity classes that interact with the laser light, and optical saturation leads to stimulated emission, causing photons to be emitted along the propagation of the laser rather than back to the telescope.

Pulsed-laser LGS systems, in the general case in which the pulses are not very short or very long, are more challenging to model, due to the dynamical processes occurring on different time scales. Experiments and modeling efforts have been reported in [27-29], among others. However, none of the theoretical studies to date have included all of the effects discussed above for the cw case, which are all relevant to pulse lengths on a $100 \mu \mathrm{s}$ time scale. In addition, a full characterization of a particular pulsed laser depends on its pulse length and pulse repetition rate and how these parameters compare to collision and relaxation rates and evolution time scales in the system, as well as the multimode and coherence characteristics of the laser.

In this paper, we report the results of physical modeling of LGS with pulsed lasers, including all of the above-mentioned effects. We focus on the TIPC pulsed laser and compute the expected photon return at the TMT site as a function of pulse length, pulse repetition rate, angular distance between the laser propagation, and the direction of the geomagnetic field. As the first field test of the pulsed laser is planned for mid-2012 at the location of the Large Zenith Telescope (LZT) operated by the University of British Columbia in Canada, we also perform simulations under the conditions at the LZT site. In addition, the photon return of the pulsed laser is compared to that obtained with a cw laser of similar transmitted power. Briefly, we find that both the cw and pulsed formats appear to be able to meet the design requirements for photon return. The $\mathrm{cw}$ and pulsed formats provide comparable photon return, with the differences at the level of the uncertainty in the model.

Results obtained from the numerical model also allow us to investigate various physical mechanisms occurring in the system that affect the photon return, often by aiding or inhibiting the generation of ground-state atomic polarization. These mechanisms can lead to interaction between seemingly unrelated parameters of the system; for example, we find that the optimal laser line width depends on the angle between the light propagation direction and the geomagnetic field direction, apparently due to the effect that each of these parameters has on the creation of atomic polarization.

The paper is organized as follows. In Section 2 the system being modeled and various physical mechanisms that can influence the photon return are discussed. In Section 3 the details of the Bloch-equation model and the numerical solver are given. Section $\underline{4}$ presents the environmental data and the geometrical optics results that are used as inputs to the model. In Section $\underline{5}$ the method of integration over the spatial extent of the beam is described. Section 6 gives the results, and conclusions are given in Section $\underline{7}$.

\section{DESCRIPTION OF THE SYSTEM}

When sodium atoms are subject to a strong circularly polarized light field resonant with the $\mathrm{D}_{2}$ transition, a number of physical processes occur that affect the efficiency at which light is spontaneously re-emitted back toward the light source.

Left-circularly polarized light resonant with the $\mathrm{D}_{2}$ a $F=$ $2 \rightarrow F^{\prime \prime}$ transition group induces optical pumping that tends to transfer the atoms to the $|F=2, m=2\rangle$ ground state 


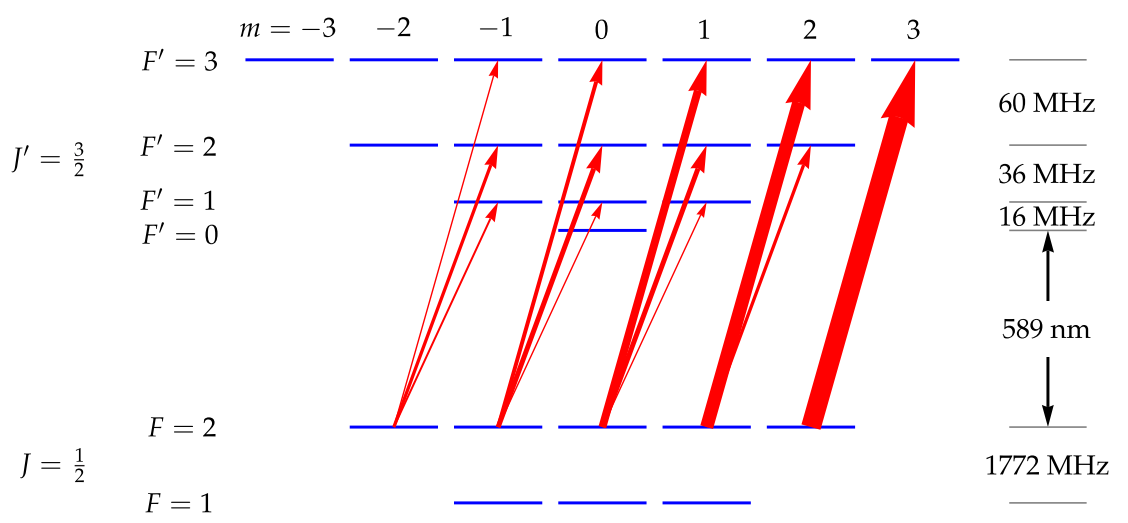

Fig. 1. (Color online) Level diagram of the $\mathrm{Na}_{2}$ line, showing transitions induced by circularly polarized light resonant with the $\mathrm{D}_{2}$ a transition. The widths of the arrows indicate the relative transition strengths.

(Fig. 1). This atomic ground-state polarization is beneficial for photon return in three ways:

1. The cycling $|F=2, m=2\rangle \rightarrow\left|F^{\prime \prime}=3, m=3\right\rangle$ transition is the strongest transition in the $\mathrm{D}_{2}$ transition group, increasing the effective absorption cross section.

2. Atoms excited on the cycling transition cannot spontaneously decay to the $F=1$ ground state, where they would no longer interact with the light.

3. Fluorescence from $\left|F^{\prime}=3, m=3\right\rangle$ is preferentially directed along the light beam, leading to an enhancement of photon flux observed at the location of the light source.

Several mechanisms work to counteract the production of atomic polarization - the relative importance of these is related to the characteristic time scale of each.

The geomagnetic field induces Larmor precession, which can act to wash out the atomic polarization. Because circularly polarized light produces atomic polarization parallel to the light propagation direction, precession will be induced if the magnetic field has a component transverse to the wave vector k (Fig. 2). A magnetic field of $0.33 \mathrm{G}$, present at the Mauna Kea site, corresponds to a Larmor precession frequency of $\sim 220 \mathrm{kHz}$ or a precession time of $4.3 \mu \mathrm{s}$, meaning that this effect is important during the $120 \mu$ s pulse produced by the TIPC laser. The detrimental effect of the magnetic field is strongly dependent on the direction of light propagation relative to the magnetic-field direction.

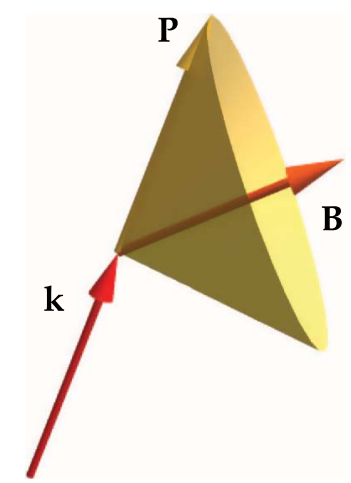

Fig. 2. (Color online) Precession of the atomic polarization $\mathbf{P}$, originally produced along the light wave vector $\mathbf{k}$, about the magnetic-field vector $\mathbf{B}$. If the polarization is continuously generated and has a lifetime longer than the Larmor precession time, it will tend to be averaged about the magnetic-field direction.
The atomic ground-state polarization is also relaxed by spin-randomization collisions, predominantly with $\mathrm{O}_{2}$ molecules [30]. The time scale for these collisions varies with the $\mathrm{O}_{2}$ density, but can be estimated to be in the 200 $5000 \mu \mathrm{s}$ range [26]. This mechanism can thus weakly affect the dynamics during a $120 \mu \mathrm{s}$ pulse. Finally, transit of the $\mathrm{Na}$ atoms through the light beam (due to the motion of the atoms or the motion of the beam itself) can also be considered to be a relaxation mechanism. The net effective atomic velocity can be estimated to be less than $370 \mathrm{~m} / \mathrm{s}$ [26], which, for a typical beam size of $20 \mathrm{~cm}$, corresponds to a millisecond time scale for transit relaxation. This effect can thus generally be neglected for the pulse lengths in question, although we include it in the numerical model for completeness.

The effective loss of atoms by spontaneous decay to the $F=1$ ground state can be directly combatted by applying "repump" light resonant with the $\mathrm{D}_{2} \mathrm{~b} F=1 \rightarrow F^{\prime}$ transition group to transfer the atoms back to the $F=2$ ground state $[13,26,30]$. As the simulation results show, this technique is vital to obtain sufficient photon return fluxes. Alleviation of the loss of atoms to the $F=1$ state also has the indirect effect of reducing the effect of the magnetic field on the return flux, as this is one of the worst effects resulting from the destruction of atomic polarization caused by Larmor precession.

Another process that tends to reduce the photon return flux for strong light fields is recoil of the atoms induced by interaction with the light. Each absorption/spontaneous emission cycle gives the atom a small momentum kick in the laser beam propagation direction; the cumulative effect of these kicks is to create holes in the Doppler distribution, depleting the atomic-velocity groups that are the most strongly resonant with the light (this is illustrated in Subsection 6.C). Velocity-changing collisions of the $\mathrm{Na}$ atoms with $\mathrm{N}_{2}$ and $\mathrm{O}_{2}$ molecules act to reequilibrate the atomic-velocity distribution, filling in the holes created by recoil. These collisions occur on a time scale of 15-300 $\mu \mathrm{s}$ [26], depending on gas density, and so can act during a single laser pulse.

\section{DESCRIPTION OF THE MODEL}

\section{A. The Bloch Equations}

In order to calculate the observed fluorescence, the evolution of the atoms is modeled using the optical Bloch equations for the atomic density matrix. The density matrix describes the magnitudes of and correlations between basis wave functions in the ensemble of $\mathrm{Na}$ atoms, in particular, the spin wave 
functions of the states involved in the $\mathrm{Na}_{2}$ transition. In order to account for atoms with different Doppler shifts, the density matrix is considered to be a function of atomic velocity along the laser beam propagation direction. (An additional degree of freedom may be included to account for the multimode structure of the laser line as discussed below.) The calculation is semiclassical in the sense that while the atoms are treated quantum mechanically, the light fields are treated classically; thus, the effect of spontaneous decay must be included phenomenologically. Because the density matrix describes all populations of, and coherences between, the 24 Zeeman sublevels making up the ground and excited states, the calculation describes, in principle, all saturation and mixing effects for essentially arbitrarily large optical and magnetic fields. (In practice, certain coherences in the system are negligible under normal experimental conditions and can be neglected in order to increase the computational efficiency.)

In order to perform numerical calculations, the velocity dependence of the density matrix is discretized to describe an appropriate number $n_{\text {v.g. }}$ of velocity groups, each with a fixed longitudinal velocity. Because coherences between atoms with different velocities can be neglected, the complete density matrix $\rho$ can be thought of as a collection of $n_{\mathrm{v} . g \text {. }}$ separate but coupled density matrices, each of dimension $24 \times 24$.

The evolution of the density matrix is given by a generalization of the Schrödinger equation:

$$
\frac{d}{d t} \rho=\frac{1}{i \hbar}[H, \rho]+\Lambda(\rho)+\beta .
$$

Here the atomic level structure and the interaction with external fields are described by the total Hamiltonian $H=H_{0}+H_{E}+H_{B}$, with $H_{0}$ the Hamiltonian for the unperturbed energy structure of the atom, $H_{E}=-\mathbf{d} \cdot \mathbf{E}$ the Hamiltonian for the interaction of the electric dipole $\mathbf{d}$ of the atom with the electric field $\mathbf{E}$ of the light, $H_{B}=-\boldsymbol{\mu} \cdot \mathbf{B}$ the Hamiltonian for the interaction of the magnetic moment $\boldsymbol{\mu}$ of the atom with the local magnetic field $\mathbf{B}$, and the square brackets denoting the commutator. The term $\Lambda$ in Eq. (1) represents phenomenological terms added to account for relaxation processes not described by the Hamiltonian. In our case these relaxation processes include spontaneous decay (omitted from the Hamiltonian due to the semiclassical approximation), collisional spin relaxation (" $S$-damping") proportional to $S^{2} \rho-S \cdot(\rho S)$ [31], where $S$ is the electronic spin-angular-momentum operator, and the exit of atoms from the light beam due to motion of the atoms and the beam (transit relaxation). In addition, there are terms included in $\Lambda$ to describe changes in atomic velocity due to collisions and light-induced recoil, as well as an effective relaxation rate for optical coherences that simulates a laser spectrum with nonnegligible bandwidth. These terms are described in more detail below. Each relaxation process described by $\Lambda$ includes a corresponding "repopulation" process, so that the trace over the density matrix for all velocity groups is conserved, corresponding to conservation of the total number of atoms. The repopulation process describing the entrance of atoms into the beam is independent of $\rho$ and so is written as a separate term $\beta$.

Velocity-changing collisions are treated as hard collisions in which the velocity of the colliding atom is rethermalized in a
Maxwellian distribution (no speed memory). The internal state of the atom is assumed to be unchanged.

Light-induced recoil is described phenomenologically by causing a fraction $v_{r} / \Delta v_{\mathrm{v} \text {.g. }}$ of the excited-state atoms in each velocity group to be transferred upon decay into the next higher velocity group. Here $v_{r}$ is the recoil velocity and $\Delta v_{\text {v.g. }}$ is the width of the particular velocity group. This model relies on the fact that $v_{r}=2.9461 \mathrm{~cm} / \mathrm{s}$ (equivalent to a Doppler shift of $50 \mathrm{kHz}$ ) is much smaller than the typical value of $\Delta v_{\mathrm{v} . \mathrm{g} .}$.

Equation (1) supplies a linear system of differential equations for the density-matrix elements, known as the optical Bloch equations. Thinking of $\rho$ as a column vector of $n_{\text {v.g. }} \times$ $24^{2}$ density-matrix elements, the Bloch equations can be written as $\dot{\rho}=A \rho+b$, where $A$ and $b$ are a matrix and a vector, respectively, that are independent of $\rho$. The vector $b$ corresponds to $\beta$ and $A$ to the rest of the right-hand side of Eq. (1).

The laser light field has a frequency component tuned near the $\mathrm{D}_{2}$ a transition group, and may have an additional "repump" component tuned near the $\mathrm{D}_{2} \mathrm{~b}$ transition group. Thus the matrix $A$ has components that oscillate at each of these frequencies. Under the rotating-wave approximation, the overall optical frequency is removed from $A$. However, the beat frequency between the two light-field components remains. This beat frequency can also be removed from the Bloch equations in our case: each frequency component interacts strongly with one transition group and very weakly with the other, so the weak coupling can be neglected for each transition. If, in addition, the small magnetic-field-induced mixing between the two hyperfine ground states is neglected, the beat frequency can be entirely removed from the evolution equations.

The fluorescent photon flux per solid angle emitted in a given direction can be found from the solution for $\rho$ as the expectation value of a fluorescence operator [32].

\section{B. Methods for Simulation of Multiple Laser Modes}

The spectrum of the TIPC laser is composed of three equally spaced modes. The proper method for including the effect of the modes in the model depends on the details of the time dependence of the spectrum, including the coherence time between the modes and the pulse-to-pulse fluctuations of the mode amplitudes. Because these details were not known with certainty, three different methods were compared in the study.

In the "coherent-modes" method, the three modes are assumed to be perfectly coherent and nonfluctuating. The atoms are subject to a field with components oscillating at each of the three mode frequencies. This is equivalent to a single field, with a frequency equal to that of the central mode, amplitude modulated with a modulation frequency equal to the mode separation. Using this model, the resulting return-flux signal exhibits beats at the modulation frequency. The width of each laser mode can be accounted for by including additional relaxation terms for the atomic optical coherences. We note that even when the effective line widths are broadened so that they overlap, the observed beats remain, because the modes are still perfectly coherent. Direct integration of the evolution equations in this situation is computationally slow, because of the rapid oscillations in the solution. Therefore, we have implemented a Floquet technique in which the explicit appearance of the rapid oscillation is removed from the evolution 
equations through expansion of the density matrix in a Fourier series. This results in a coupled system of equations for the relatively slowly evolving Fourier coefficients. These equations can be efficiently integrated to find the time dependence of the lowest few Fourier harmonics.

In the "mode-hopping" method, the laser frequency is assumed to fluctuate between the three mode frequencies on a time scale much shorter than the pulse length. This is accomplished by writing density matrices describing "regions," each subject to one of the laser modes, and allowing atomic transit between the regions. The transit rate corresponds to the width of each laser mode. In this model, the optical coherences are assumed to be destroyed upon transit between regions, meaning that the laser modes can be considered to be effectively incoherent. Thus no beats are observed in the return flux.

In the "pulse-averaging" method, large pulse-to-pulse fluctuations in the relative mode amplitudes are assumed, so that during each pulse the laser power is present on only one of the three modes, with equal probability. This is accomplished by running the simulation three times, once with the light frequency equal to each mode frequency, and averaging the results. The modes are effectively incoherent with this method.

\section{The Numerical Solver}

The density-matrix evolution equations are generated using the LGSBloch package for Mathematica, which is based on the Atomic Density-Matrix package written by Rochester [33]. The system of ordinary differential equations is solved using a code written in $\mathrm{C}$ and based on the open-source ODE solver CVODE, from the SUNDIALS package [34]. This solver addresses the stiffness of the system using the implicit backward differentiation formulas (BDFs) [35]. These methods require the solution of a linear system of equations at each step of the solver; these solutions are accomplished with a preconditioned Krylov method. (In such a method an initial guess is improved by minimizing the residual over a subspace with dimension much smaller than that of the full system [35].) The rate of convergence of the method is increased by premultiplication with a block-diagonal preconditioner (approximate inverse of $A$ ), obtained by setting all terms that connect density-matrix elements from different velocity groups to zero, and then inverting the block for each velocity group. For the mode-hopping multimode method, the transit terms that couple the density matrices for the three modes are also included in the preconditioner, using an operator splitting technique that inverts these terms separately. For the coherentmodes Floquet technique, the terms for all of the included coupled Fourier coefficients are included in the block for each velocity group.

\section{INPUT DATA}

A. Standard Parameters and Environmental Conditions The standard parameters used in the model for the TIPC laser are given in Table 1 . Environmental parameters and parameters specific to the Mauna Kea and LZT sites are listed in Table 2. The sodium density as a function of altitude is assumed to be described by a Gaussian function centered at $93 \mathrm{~km}$ with a full width at half maximum (FWHM) of $8 \mathrm{~km}$. This is a rough estimate, as the measured density profile at any given time may deviate significantly from a Gaussian. However, our modeling indicates that the photon return
Table 1. Standard Parameters for the TIPC Pulsed Laser

$\begin{array}{lc}\text { Average laser power } & 20 \mathrm{~W} \\ \text { Average projected laser power } & \\ \text { Mauna Kea } & 12 \mathrm{~W} \\ \text { LZT } & 18.6 \mathrm{~W} \\ \text { Polarization } & \text { circular } \\ \text { Number of modes } & 3 \\ \text { Mode spacing } & 150 \mathrm{MHz} \\ \text { Pulse length } & 120 \mu \mathrm{s} \\ \text { Repetition rate } & 800 \mathrm{~Hz}\end{array}$

has only a weak dependence on the width of the density profile: increasing the assumed width to $11 \mathrm{~km}$ leads to a reduction in photon return of about $1 \%$. For each site, the temperature and gas densities as a function of altitude in the sodium layer were obtained from the MSIS-E-90 Atmosphere Model [36]; for the Mauna Kea site, these values, along with the assumed $\mathrm{Na}$ density profile, are plotted in Fig. 3. Collision rates and the Doppler width as a function of altitude are derived from these data and the assumed collision cross sections are given in Table 3 .

\section{B. Laser Spot Image Model}

To find the expected laser intensity profile, a geometrical optics simulation was performed. The LLT has a diameter of $0.4 \mathrm{~m}$ and is nominally focused (absent diffraction and turbulence effects) at a range of $125 \mathrm{~km}$. The Gaussian laser beam has a waist diameter ( $e^{-2}$ of the peak intensity) of $0.24 \mathrm{~m}$ at the LLT pupil.

We computed the expected laser intensity profile at ranges of $85,95,105,115$, and $125 \mathrm{~km}$. This was accomplished by modeling the propagation of the $589 \mathrm{~nm}$ laser light from the launch telescope for several instantiations of a single turbulence screen assumed to be located close to the LLT. The turbulence screen was modeled with a von Karman power spectral density, an outer scale of turbulence [37] set to $30 \mathrm{~m}$, and for various turbulence strengths characterized by the Fried lengths $r_{0}=0.10,0.15,0.20,0.25$, and $0.30 \mathrm{~m}$. The TIPC geometrical beam quality is characterized by a broadening factor of $M^{2}=1.2$. This $M^{2}$ factor is achieved in the light propagation model by introducing a slight coma aberration

Table 2. Standard Parameters for Mauna Kea and LZT sites ${ }^{a}$

\begin{tabular}{lcc}
\hline & Mauna Kea & LZT \\
\hline Latitude & $19.8^{\circ}$ & $49.3^{\circ}$ \\
Longitude & $155.5^{\circ}$ & $122.6^{\circ}$ \\
Observatory altitude & $4050 \mathrm{~m}$ & $395 \mathrm{~m}$ \\
B-field & $0.334 \mathrm{G}$ & $0.525 \mathrm{G}$ \\
Na Larmor frequency & $234 \mathrm{kHz}$ & $367 \mathrm{kHz}$ \\
B-field zenith angle & $126^{\circ} 18^{\prime}$ & $160^{\circ} 30^{\prime}$ \\
B-field azimuthal angle & $9^{\circ} 45^{\prime}$ & $16^{\circ} 57^{\prime}$ \\
Atmospheric transmission (zenith) & 0.84 & 0.8 \\
Fried length (zenith) & $21 \mathrm{~cm}$ & $5 \mathrm{~cm}$ \\
Na column density & \multicolumn{2}{c}{$4 . \times 10^{13} \mathrm{~m}^{-2}$} \\
Na centroid altitude & \multicolumn{2}{c}{$83 \mathrm{~km}$} \\
Na layer thickness & \multicolumn{2}{c}{$8 \mathrm{~km}$} \\
\hline
\end{tabular}

${ }^{a}$ The Fried length is the size of the circular aperture within which the variance of the wavefront phase aberrations is less than 1 radian [24]. 


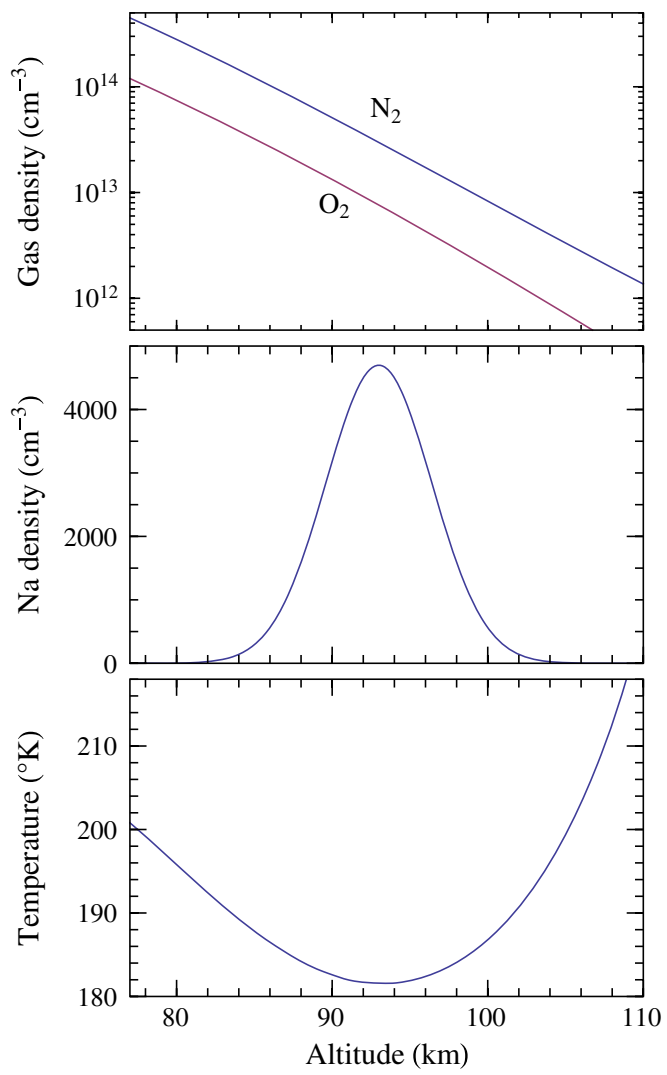

Fig. 3. (Color online) Temperature and $\mathrm{O}_{2}$ and $\mathrm{N}_{2}$ gas densities as a function of altitude obtained from the MSIS-E-90 Atmosphere Model for the Mauna Kea site, along with the assumed Na density profile. (Note that deviations of the actual Na density profile from a Gaussian are often quite large.)

in the LLT mirror and a total wavefront error of $76.5 \mathrm{~nm}$ rms. In addition, because the LLT mirror has a fixed focal distance of $f_{L}=125 \mathrm{~km}$, the laser intensity beam profiles at altitudes lower than $125 \mathrm{~km}$ also include the effect of a focus error modeled as $r^{2} \Delta h /\left(2 f_{L}^{2}\right)$, where $r$ is the laser beam radius and $\Delta h$ is the distance from the LGS spot to the $125 \mathrm{~km}$ focal distance.

The beam profiles generated through the process above were interpolated as needed for intermediate values of $r_{0}$ used for simulations at the Mauna Kea site. At the LZT site, the atmospheric turbulence is stronger, with the median turbulence conditions better characterized by an $r_{0}$ of $0.05 \mathrm{~m}$. In this case, the laser beam profile was extrapolated linearly down to $r_{0}=0.05 \mathrm{~m}$ from the profiles available for larger values of $r_{0}$.

The LGS spot images are sampled at 0.121 " and are 64 pixels across. An example of the beam profile is given in Fig. 4(a). Fitting the narrower dimension of the beam profiles with a Gaussian function yields angular sizes ranging from 0.68 " to 0.37 " FWHM for $r_{0}=0.05$ to $0.3 \mathrm{~m}$.

Table 3. Estimated Values for Cross Sections $\underline{a}$

\begin{tabular}{cc}
\hline$\sigma_{\mathrm{Na}-\mathrm{N}_{2}}$ & $7.15 \times 10^{-15} \mathrm{~cm}^{2}$ \\
$\sigma_{\mathrm{Na}-\mathrm{O}_{2}}$ & $7 \times 10^{-15} \mathrm{~cm}^{2}$ \\
$\sigma_{\mathrm{Na}-\mathrm{O}_{2}}^{S}$ & $2.5 \times 10^{-15} \mathrm{~cm}^{2}$ \\
\hline${ }^{a} \mathrm{See}[26]$ and references therein; note that the factor of \\
$1 / 2$ described in [26] is incorporated into $\sigma_{\mathrm{Na}-\mathrm{O}_{2}}^{S} \cdot$
\end{tabular}

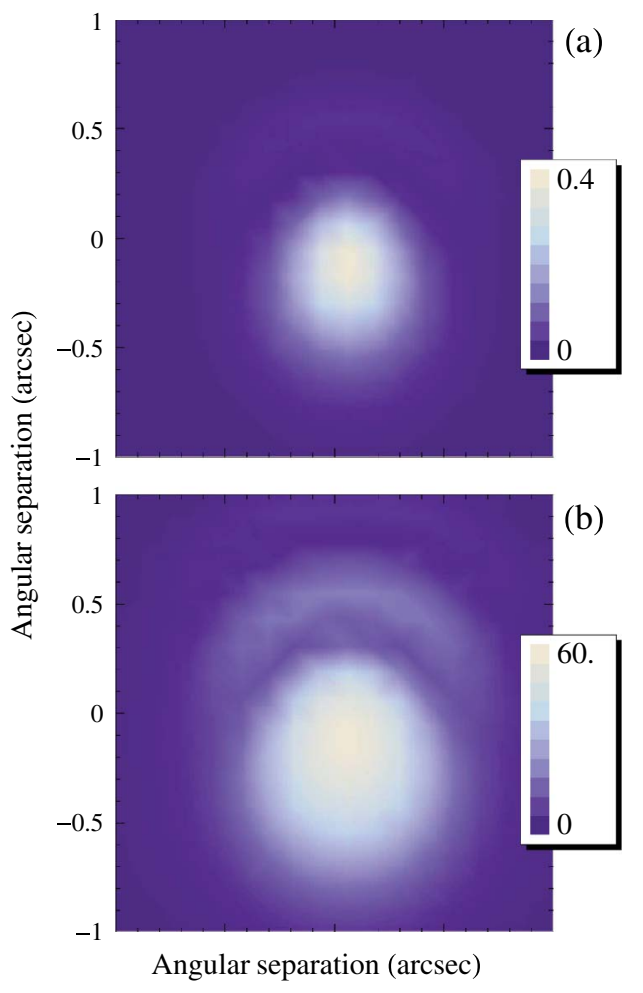

Fig. 4. (Color online) (a) Interpolated beam profile from geometrical optics model, given in terms of the fraction of power in each pixel. The peak value is the Strehl ratio. (b) Return flux from each pixel in $10^{3}$ photons $/ \mathrm{s} / \mathrm{m}^{2}$.

\section{SPATIAL INTEGRATION}

Integration of the observed return flux over the spatial extent (profile and path length) of the laser beam is accomplished in four steps, using a method similar to that of [38]:

1. Construct table of return flux versus intensity and altitude.

2. For each pixel in the modeled beam profile, propagate light through sodium layer using the table, keeping track of absorption.

3. Integrate return flux over path length.

4. Sum over pixels.

We first generate a table of return fluxes as a function of light intensity and altitude. Parameters that change with altitude, plotted in Fig. 3, are $\mathrm{N}_{2}$ and $\mathrm{O}_{2}$ density (affects collision rates), $\mathrm{Na}$ density (multiplicative factor), and temperature (weakly affects collision rates and Doppler width). Sodium density is modeled by a Gaussian with integrated column density $4 \times 10^{13} \mathrm{~m}^{-2} \cdot \mathrm{N}_{2}$ and $\mathrm{O}_{2}$ density and temperature are obtained from the MSIS-E-90 Atmosphere Model [36].

Both the return flux $\Phi(I, z)$ and total fluorescence into $4 \pi$, $\Phi_{\text {tot }}(I, z)$, are obtained from the model. Return values from the model are multiplied by the $\mathrm{Na}$ density and area of a 0.12 " spot (the size of a pixel in the beam profile) at a given altitude. This results in values of fluxes per unit path length $d \ell$.

Light power in the uplink beam is absorbed as it travels through the sodium layer, and reradiated as fluorescence in all directions. For a sub-beam with angular size equal to one pixel in the beam profile, the power absorbed per unit path length $d \ell$ is $\hbar \omega d \Phi_{\text {tot }}(I, z) / d \ell$, with $I=P(\ell) /\left(\theta_{p} \ell\right)^{2}$ and $z=\ell / X$, where $P(\ell)$ is the power at distance $\ell, \theta_{p}$ is 
the angular size of one pixel, $X=\sec \zeta$ is the airmass ( $\zeta$ is the zenith angle) [39], and $\hbar \omega$ is the energy of one photon. Thus the power along the optical path is given by the propagation equation

$$
d P(\ell) / d \ell=-\hbar \omega d \Phi_{\text {tot }}\left(P(\ell) /\left(\theta_{p} \ell\right)^{2}, \ell / X\right) / d \ell
$$

We integrate this equation for a range of initial light powers, chosen to cover the range obtained using the given beam profile and the average laser power.

For the transmission of the return flux through the sodium layer, we assume linear absorption and so use the curve obtained from Eq. (2) in the low power limit, denoted by $T_{\text {lin }}(\ell)$. The observed return flux into a detector of unit area per unit path length emitted from a pixel-sized sub-beam at a distance $\ell$ is then given by

$$
d \Phi_{\text {obs }}\left(P_{0}, \ell\right) / d \ell=\frac{T_{a}^{X}}{\ell^{2}} T_{\operatorname{lin}}(\ell) d \Phi\left(P(\ell) /\left(\theta_{p} \ell\right)^{2}, \ell / X\right) / d \ell
$$

where $P(\ell)$ depends on the initial power in the sub-beam, $P_{0}$, and $T_{a}$ is the atmospheric transmission at zenith.

Integrating $d \Phi_{\text {obs }}\left(P_{0}, \ell\right) / d \ell$ over the path length, we find the observed flux from each sub-beam as a function of the initial power, $\Phi_{\text {obs }}\left(P_{0}\right)$. We then sum the return flux from all of the pixels in the beam profile. An example of the return flux profile is shown in Fig. 4(b), the intermediate steps in the integration procedure are illustrated in Subsection 6.D.

\section{RESULTS}

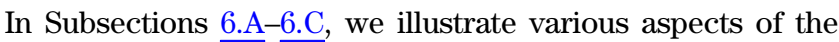
signal using the return flux calculated from a particular location in the sodium layer, i.e., not integrated over the spatial extent of the beam. Subsection 6.D illustrates the steps taken in the spatial integration procedure, Subsection 6 .E presents the integrated return-flux results, and Subsection $6 . \mathrm{F}$ presents plots that relate to optimization of the pulse length, repump power fraction, and laser line width.

\section{A. Time Dependence}

Examples of the time dependence of photon flux return during a $120 \mu$ s square pulse using the three different methods for treating the multimode laser spectrum (coherent modes, mode-hopping, and pulse averaging) are shown in Fig. 5. In Fig. 5(a), no repump light is applied. Following the initial rapid rise in flux at the beginning of the pulse, the flux begins to fall off. This is primarily due to pumping to the $F=1$ ground state, as can be seen by diverting some of the laser power into the repump light beam [Fig. $\underline{5(\mathrm{~b})}$ ], which counteracts most of the falloff. As throughout, unless otherwise noted, we take $10 \%$ of the total laser power to be repump light. We also assume that the repump light is generated by amplitude modulation of the $\mathrm{D}_{2}$ laser field, meaning that an additional $10 \%$ of the laser power is lost into the additional low-frequency sideband that is produced. Unless otherwise noted, the repump light is taken to be of the same polarization as the main light field. The effect of the repump light is examined in more detail in Subsection 6.C.

The rapid beats present when using the coherent-mode treatment can be seen more clearly in Fig. $\underline{6}$, which shows the initial part of the pulse.

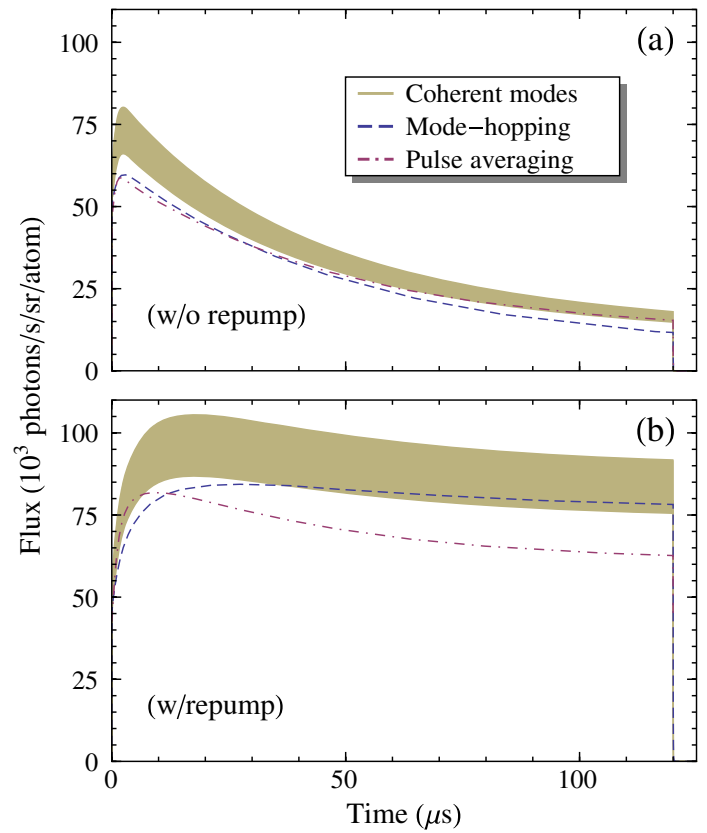

Fig. 5. (Color online) Instantaneous return flux during a single pulse as a function of elapsed time, using three different multimode treatments, both with no repump light and with $10 \%$ repump. Example plots are for Mauna Kea, zenith angle zenith angle $=30^{\circ}$, azimuth angle $=190^{\circ}, I_{\mathrm{avg}}=27.5 \mathrm{~W} / \mathrm{m}^{2}$. The oscillations present in the coherent-mode treatment are too rapid to be distinguished on this time scale, and so appear as solid bands.

\section{B. Pulse Shape}

The results of Fig. $\underline{5}$ are calculated assuming a perfectly square pulse shape. A measurement of the actual pulse shape produced by the TIPC laser is shown in Fig. 7. This shape differs from a square pulse in that there are finite rise and fall times, and also short spikes in the initial part of the pulse. To investigate the effect of these differences, we defined an approximate analytical model for the measured pulse shape. The model represents the initial spikes as rapid exponentially decaying oscillations. While not a completely faithful reproduction of the measured pulse shape, the accuracy of this representation should be enough to indicate whether the initial spikes have the potential to cause a significant deviation from the results obtained using an ideal square pulse.

To model the measured pulse shape, we used a functional form with the pulse rise given by

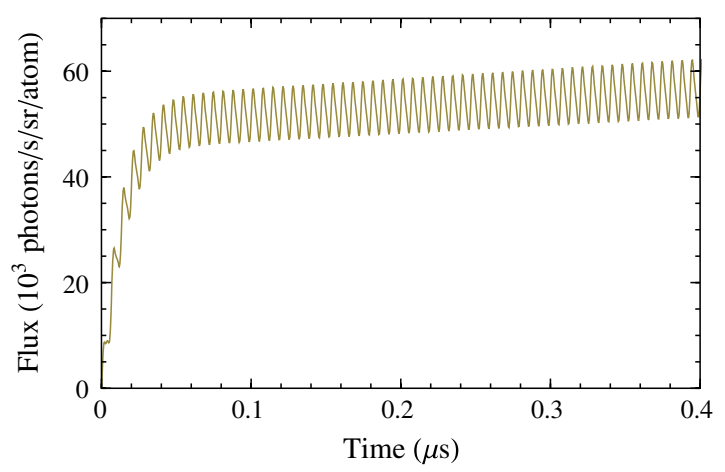

Fig. 6. (Color online) An expanded view of the initial part of the coherent-modes plot from Fig. 5(b). The oscillations are due to interference between the laser modes. 


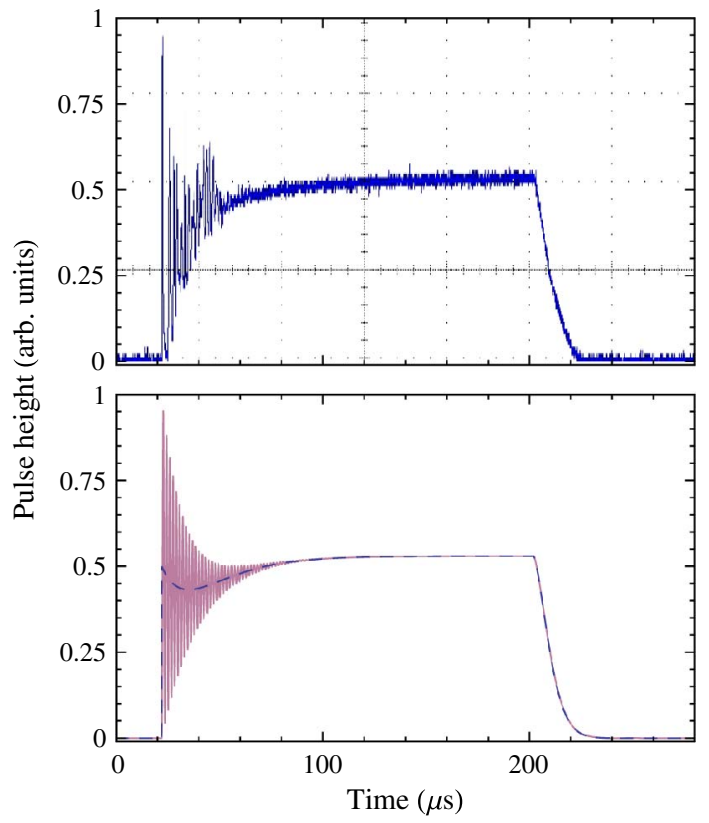

Fig. 7. (Color online) Laser pulse shapes: (top) the supplied measured pulse shape; (bottom) the pulse-shape model described in the text (solid line) and the same model with the initial oscillations averaged out (dashed line). The parameters used here are $t_{0}=22 \mu \mathrm{s}$, $\tau_{r}=19 \mu \mathrm{s}, a_{\mathrm{osc}}=1.9, \tau_{\mathrm{osc}}=12 \mu \mathrm{s}, \omega=2 \pi \times 0.6 \mathrm{MHz}, t_{1}=202 \mu \mathrm{s}$, $\tau_{f}=9.6 \mu \mathrm{s}$, and $p=1.5$. The overall amplitude is scaled to match the measurement. The nominal pulse length is $t_{1}-t_{0}=180 \mu \mathrm{s}$, longer than the standard pulse length of $120 \mu$ s used in the simulations.

$$
1-e^{-\left(t-t_{0}\right) / \tau_{r}}+a_{\mathrm{osc}} e^{-\left(t-t_{0}\right) / \tau_{\mathrm{osc}}} \sin ^{2}(\omega t / 2),
$$

where $t_{0}$ is the pulse start time, $\tau_{r}$ is the rise time, $a_{\mathrm{osc}}$ is the amplitude of the initial oscillations, $\tau_{\text {osc }}$ is the decay time of the oscillations, and $\omega$ is the oscillation frequency. The pulse fall is somewhat faster than exponential, and so is modeled by

$$
\exp \left(-\left[\left(t-t_{1}\right) / \tau_{f}\right]^{p}\right)
$$

where $t_{1}$ is the time at the beginning of the fall, $\tau_{f}$ is the nominal fall time, and $p \approx 1.5$.

Appropriate parameters were chosen by eye to match the supplied measured plot, as shown in Fig. 7. Also shown in the figure is a pulse shape obtained by averaging out the initial oscillations of the formula (4).

The calculated responses to a square pulse, a pulse with initial oscillations, and a pulse with the initial oscillations averaged out are shown in Figs. 8(a), 8(b), and 8(c), respectively. The pulses are each $120 \mu \mathrm{s}$ from the beginning of the pulse to the start of the fall, with the other pulse-shape parameters chosen to match those given in Fig. 7 . The three types of pulses are each normalized to have the same total pulse energy. A single-mode laser is assumed for these plots.

The time-averaged flux from the more realistic pulse is higher than that from the square pulse, apparently because the pulse energy is spread out over a longer effective time due to the pulse fall time. Averaging out the initial oscillations has almost no effect on the time-averaged flux.

These results indicate that the deviations of the measured pulse shape from a square pulse have an insignificant effect on the photon return, given the current uncertainty in the

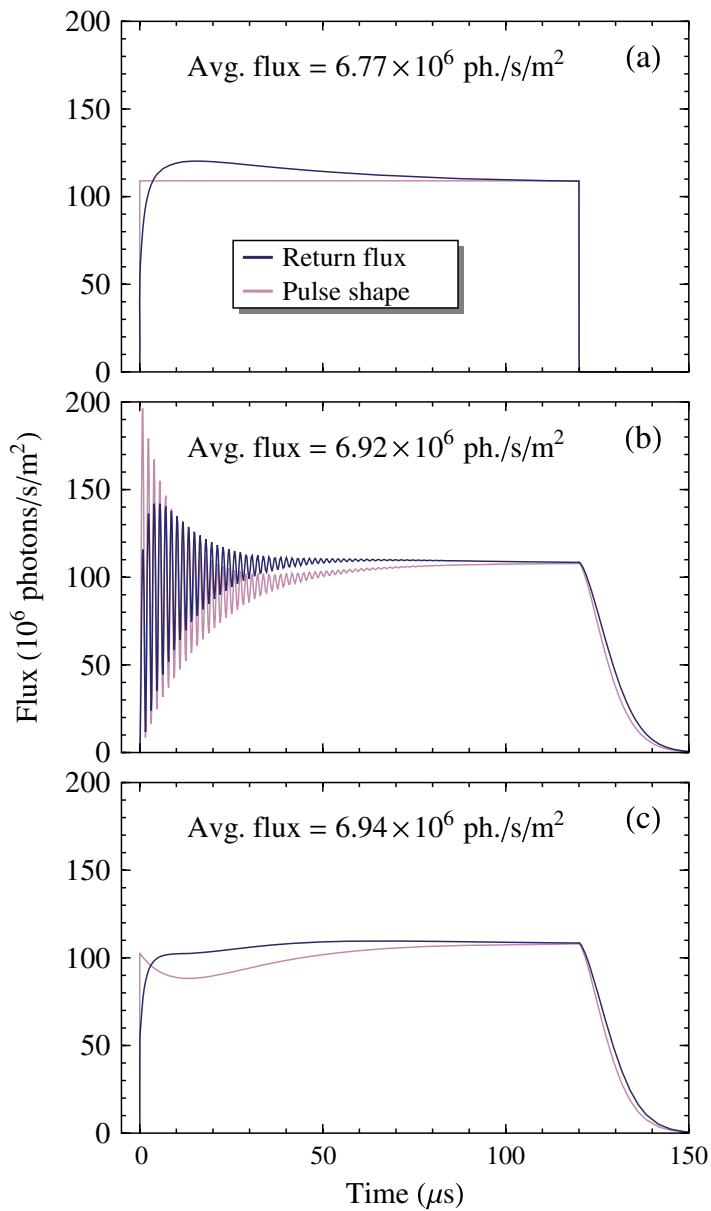

Fig. 8. (Color online) Return flux in response to three different pulse shapes: (a) ideal square pulse, (b) pulse with initial oscillations and finite rise and fall times, and (c) pulse as in (b) but with initial oscillations averaged out. A single-mode laser is assumed. The nominal pulse lengths are $120 \mu \mathrm{s}$; the other pulse-shape parameters are as given in the caption to Fig. 7 .

environmental and laser parameters. We therefore will use an ideal square pulse in the following simulations.

\section{Atomic-Velocity Dependence}

The return flux as a function of the atomic-velocity group near the beginning of the laser pulse is shown in Fig. 9. The response to the three laser modes is seen, with the shape of each peak determined by the upper-state hyperfine structure of the $\mathrm{D}_{2}$ line.

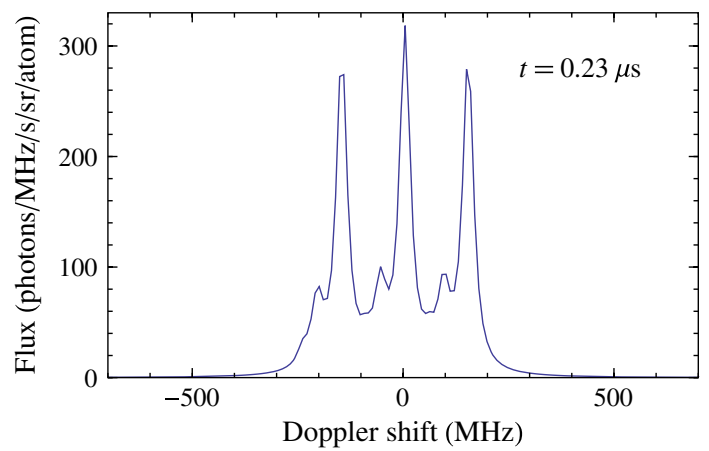

Fig. 9. (Color online) An example of return flux as a function of the longitudinal atomic velocity (given in terms of the corresponding Doppler shift), at a time near the beginning of a laser pulse. 


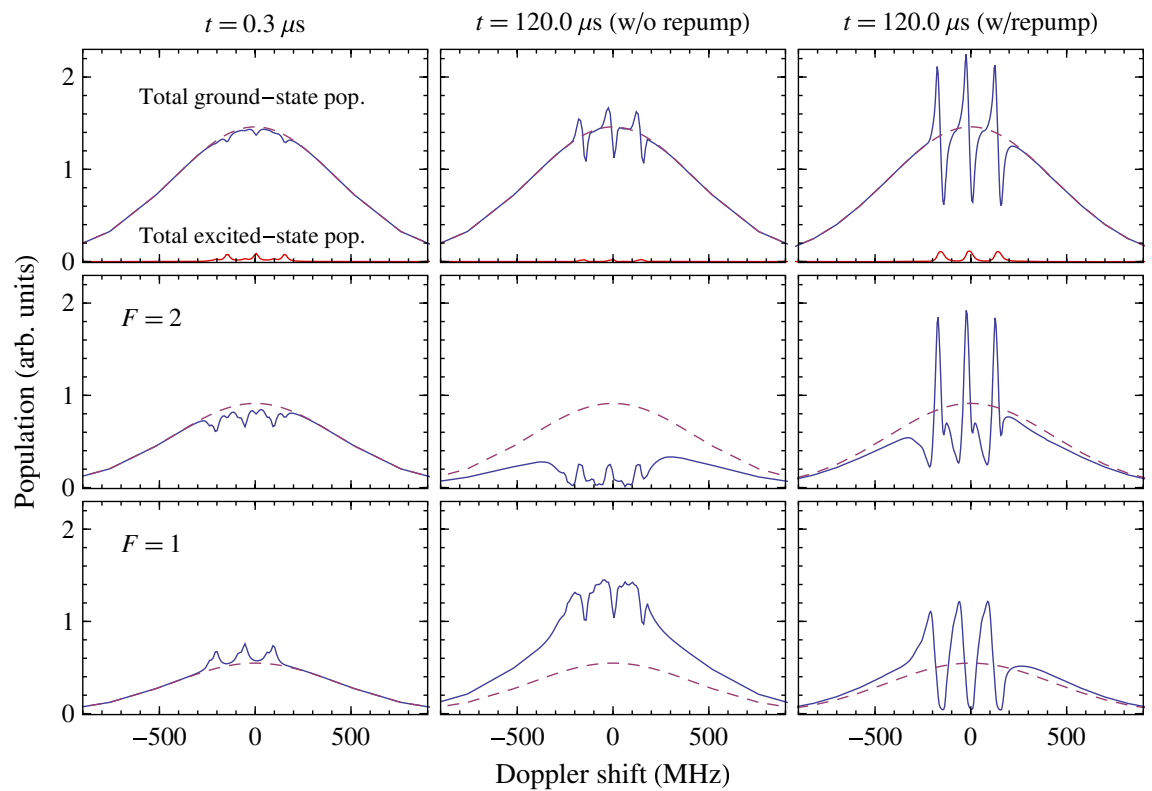

Fig. 10. (Color online) Populations of the $F=1$ ground state (bottom row), $F=2$ ground state (middle row), and total ground-state and upperstate populations (top row), as a function of longitudinal atomic velocity for the mode-hopping method. Populations are shown for times near the beginning of the pulse (left column), at the end of a pulse without repump light (middle column), and at the end of a pulse with repump light (right column), superimposed over the unperturbed equilibrium populations (dashed lines).

To study the effect of various mechanisms on the dynamics of the atomic system, it is helpful to plot atomic populations as a function of velocity group at various times during the pulse. Figure 10 shows populations of the $F=1$ ground state (bottom row), $F=2$ ground state (middle row), and total

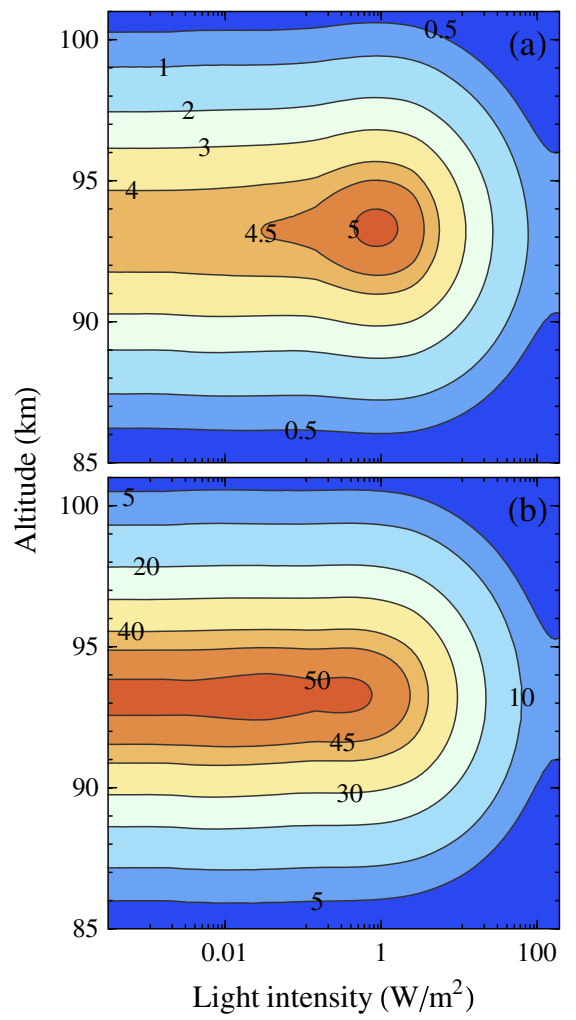

Fig. 11. (Color online) (a) Return flux $d \Phi(I, z) / d \ell / I$ in units of $10^{9}$ photons $/ \mathrm{s} / \mathrm{sr} / \mathrm{m} /\left(\mathrm{W} / \mathrm{m}^{2}\right)$ as a function of light intensity and altitude. (b) Total flux $d \Phi_{\text {tot }}(I, z) / d \ell / I$ in units of $10^{9}$ photons $/ \mathrm{s} / \mathrm{m} /\left(\mathrm{W} / \mathrm{m}^{2}\right)$, used to calculate optical absorption in the sodium layer. ground-state and upper-state populations (top row), as a function of longitudinal atomic velocity using the mode-hopping treatment of the laser modes. The left-hand column shows the populations near the beginning of the pulse. A small amount of the total population has been transferred from the ground state to the upper state, and some atoms have been transferred from the $F=2$ ground state to the $F=1$ ground state by optical pumping. The middle column shows the populations at the end of the pulse if no repump light is used. In this case, the majority of the $F=2$ atoms have been pumped into the $F=1$ ground state, where they do not interact with the laser light. As a result, the number of atoms in the excited state (and hence the photon return flux) is greatly reduced. The hole-burning effect of atomic recoil can also be seen in the total ground-state population. The right-hand column shows what happens if repump light is used. In this case, the population of the $F=2$ state is largely preserved, as atoms pumped to the $F=1$ state are repumped to the $F=$ 2 state. When repump light is applied, the effect of recoil becomes much more dramatic, as each atom is able to interact with the light many more times during the pulse.

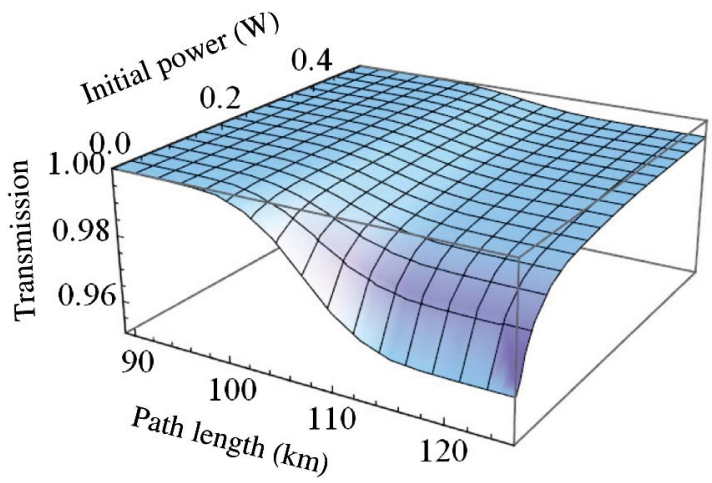

Fig. 12. (Color online) Transmission through the sodium layer as a function of path length and initial light power in a pixel-sized beam. 


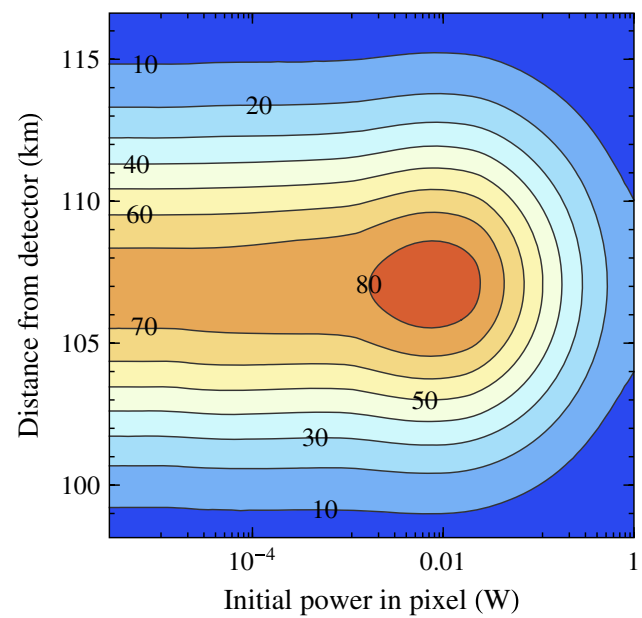

Fig. 13. (Color online) Normalized observed return flux per $d \ell$ from a pixel-sized sub-beam, $d \Phi_{\text {obs }}\left(P_{0}, \ell\right) / d \ell / P_{0}$, in units of photons $/ \mathrm{s} / \mathrm{m}^{2} / \mathrm{m} / \mathrm{W}$.

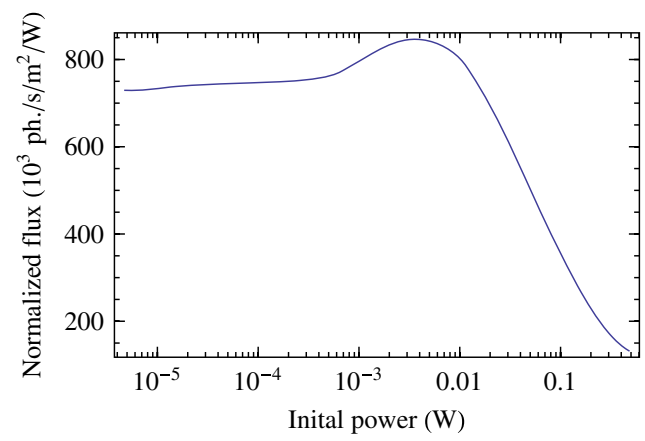

Fig. 14. (Color online) Total observed normalized return flux $\Phi_{\text {obs }}\left(P_{0}\right) / P_{0}$ from a pixel-sized beam as a function of initial launched light power in that pixel.

\section{Spatial Integration}

Here we illustrate the steps taken to perform the integration of the photon return over the spatial extent of the beam. First, we run the model a number of times assuming a 0.12 " pixelsized spot to construct a table of the return flux $d \Phi(I, z) / d \ell$ as a function of altitude and intensity [Fig. 11(a)]. At the same time, we also construct a table of the total fluorescence [Fig. 11(b)], which is used to calculate the resonant absorption of light in the sodium layer (Fig. 12).

Accounting for resonant absorption on the uplink and downlink, we can then construct a table of observed fluorescence at the detector per unit path length in the sodium layer, $d \Phi_{\text {obs }}\left(P_{0}, \ell\right) / d \ell$, as a function of distance and initial power in each pixel (Fig. 13). Integrating over distance, we find the total return per pixel as a function of initial power, $\Phi_{\text {obs }}\left(P_{0}\right)$ (Fig. 14). As seen in Fig. 14, the efficiency of return is constant for low power (the linear regime), begins to rise as power is increased - due to the beneficial effects of optical pumpingand then falls off due to saturation effects, pumping to the $F=1$ state, and recoil.

We then take the beam profile obtained from the geometrical optics simulation with the appropriate adjusted Fried parameter [Fig. 4(a)], and apply $\Phi_{\text {obs }}\left(P_{0}\right)$ to each pixel to obtain the return-flux spot profile [Fig. 4(b)]. Because of the saturation effect, the central peak of the return-flux profile is compressed relative to that of the beam profile, increasing the relative prominence of the dimmer features due to the astigmatism seen at the top of the profile.

Summing over the pixels of the photon return-flux profile gives the final calculated value for the total return flux.

\section{E. Beam-Integrated Return Flux}

\section{Return Flux as a Function of Zenith Angle}

The total return flux integrated over the spatial extent of the beam is shown in Fig. 15 as a function of zenith angle for pulsed light, using each of the three multimode methods, and also for narrow-band cw light. Positive zenith angles
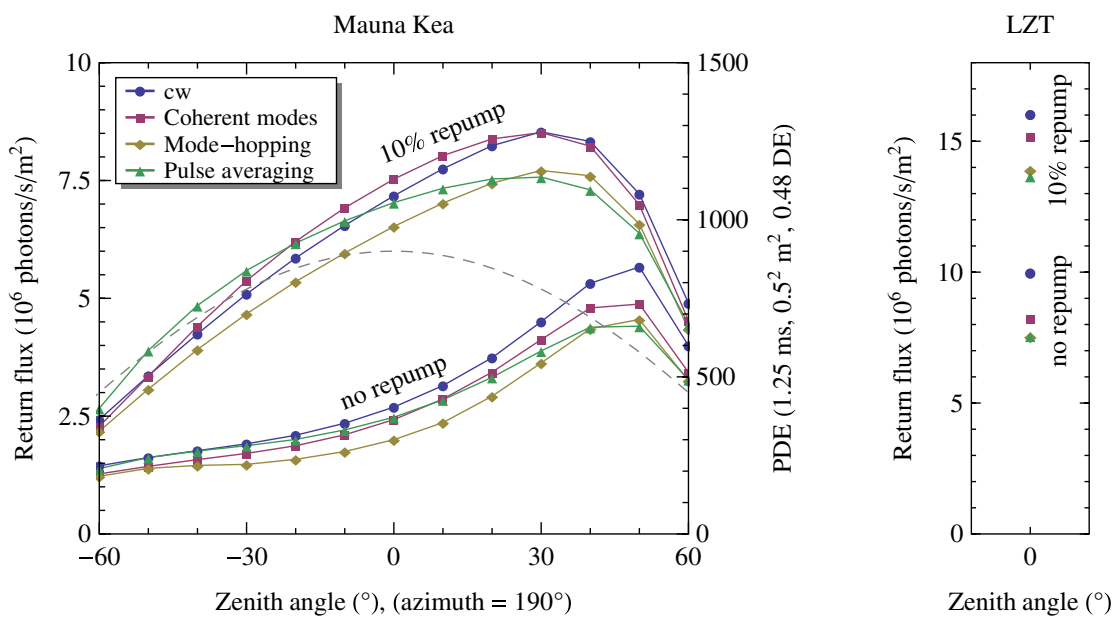

Fig. 15. (Color online) Beam-integrated return flux for Mauna Kea (as a function of zenith angle) and LZT (at zenith), for pulsed light using each of the three multimode methods and for narrow-band cw light. Results are given for both no repump light and for $10 \%$ of the light power in the repump beam. The vertical axis on the left side is in units of $10^{6}$ photons $/ \mathrm{s} / \mathrm{m}^{2}$, while the axis on the right side of the plot for Mauna Kea shows photodetected electrons (PDE) assuming a collection time of $1.25 \mathrm{~ms}$, collection area of $0.5 \mathrm{~m}^{2}$, and $48 \%$ detection efficiency. The dashed gray line shows the design requirement for 900 PDEs at zenith, combined with the nominal dependence on the inverse of the airmass $X$ expected from purely geometrical considerations. (The optical path length in the sodium layer increases with $X$, while the detector collection solid angle falls as $X^{-2}$.) The higher return at the LZT site is primarily due to the higher throughput of the laser-launch system and the different direction of the local geomagnetic field compared to that at the Mauna Kea site. 
correspond to $190^{\circ}$ azimuth, while negative zenith angles correspond to $10^{\circ}$ azimuth. Results are shown both for no repump light and for the standard repump fraction (i.e., $10 \%$ of the light power in the repump beam, $80 \%$ of the laser power in the $\mathrm{D}_{2} \mathrm{a}$ light, and $10 \%$ lost).

The three methods for modeling the laser modes give results that vary by $\sim 15 \%$; this may be taken as an indicator of the level of uncertainty in the model due to incomplete knowledge of the characteristics of the laser. (By running calculations with finer grid spacings and stricter tolerances, we have found that uncertainties due to numerical approximations in the model are below 5\%.) At this level of uncertainty, the narrow-band cw light provides approximately the same photon return as the pulsed format. These results indicate that both pulsed and cw light have the potential to satisfy the TMT design requirements as long as repump light is used, and will likely not satisfy the requirements if repump is not used.

\section{Reversed-Polarization Repump}

For technical reasons, it may be easier to produce repump light with opposite polarization to that of the main $\mathrm{D}_{2}$ a light. Figure 16 shows the effect of reversing the polarization of the repump light. Results are shown for a pulsed laser with no repump, with $10 \%$ repump, and with $10 \%$ repump with opposite circular polarization to the $\mathrm{D}_{2}$ a light, using the pulseaveraging multimode treatment. Using opposite circular polarization for repumping incurs a $5-10 \%$ penalty, depending on zenith angle, relative to repumping with the same polarization. Because this is a comparison of the same laser format and multimode model with itself, an observed difference at this level may be numerically significant.

\section{Dependence on Spot Size for LZT site}

To find the dependence of return flux on spot size for the LZT site, the beam profile was scaled proportionally from the nominal size 0.68 " FWHM obtained from the geometrical optics model using the standard Fried parameter $r_{0}=0.05 \mathrm{~m}$. When varying the beam size from $1 / 2$ to three times the standard beam size, the return flux varies from about $25 \%$ below to $25 \%$ above the value obtained with the standard size (Fig. 17).

\section{F. Optimization}

In this subsection, we plot the integrated return flux for the Mauna Kea site as a function of zenith angle and various other parameters for use in optimization. Results for pulsed and

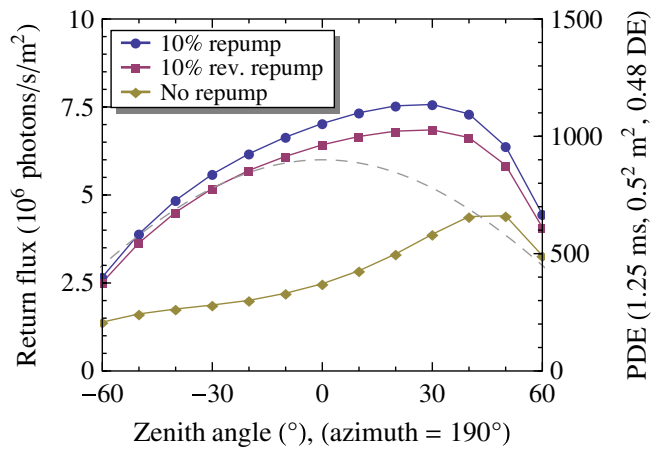

Fig. 16. (Color online) Beam-averaged return flux and PDE for a pulsed laser without repump, with $10 \%$ repump, and with $10 \%$ repump with opposite circular polarization as the $\mathrm{D}_{2}$ a light.

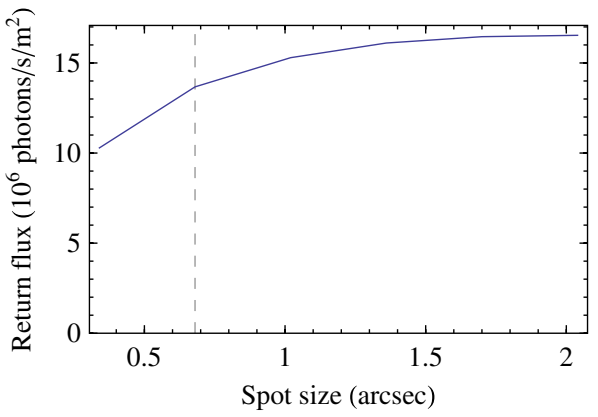

Fig. 17. (Color online) Return flux as a function of spot size (FWHM) at the LZT site, using the pulse-averaging method. The dashed gray line shows the spot size obtained from the geometrical optics model using the standard Fried parameter $r_{0}=0.05 \mathrm{~m}$. The beam profile was directly scaled proportionally smaller and larger to obtain the results for this plot.

cw lasers are given; for the pulsed format, we use the pulseaveraging multimode treatment.

Figure 18 shows a contour plot of return flux in units of $10^{6}$ photon $/ \mathrm{s} / \mathrm{m}^{2}$ as a function of zenith angle and pulse length, holding the repetition rate and the average laser power constant. The standard amount of repump light (of the same polarization as the $\mathrm{D}_{2}$ a light) is used. The plot runs over the range of pulse lengths that may be accessible to the TIPC laser. In this range, the plot shows that longer pulse lengths are generally better.

Figure 19 shows a contour plot of return flux as a function of zenith angle and repump power percentage for the pulsed and cw cases. For Mauna Kea, it appears that 10\% repump is optimal for both pulsed and cw light.

Figure 20 shows a contour plot of return flux as a function of zenith angle and the width of each laser mode for the pulsed and cw cases. The standard amount of repump light is used. The optimal width depends on the zenith angle, or more precisely, the angle between the light propagation direction and the magnetic field. When this angle is small, a broader line width is advantageous, apparently because the reduced effect of Larmor precession means that less light intensity per velocity group is required to produce beneficial atomic polarization. When the angle is larger, narrower laser lines are optimal. For large angles, the optimum line width is reasonably consistent with the value of $\sim 20 \mathrm{MHz}$ obtained from the formula given in [26] for the cw case. From these results,

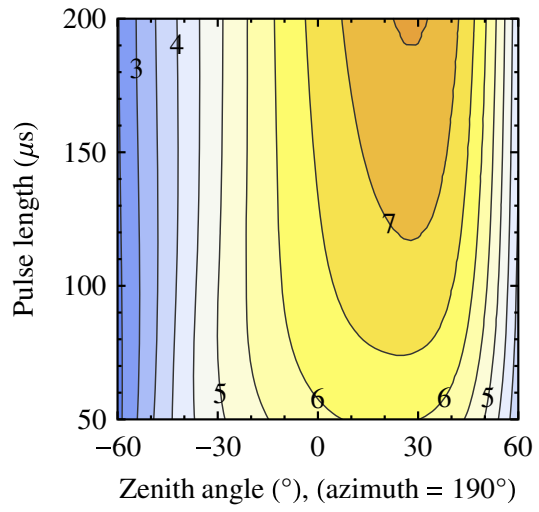

Fig. 18. (Color online) Return flux in units of $10^{6}$ photon $/ \mathrm{s} / \mathrm{m}^{2}$ as a function of zenith angle and pulse length for the Mauna Kea site. The repetition rate and average laser power are held constant as the pulse length is varied. 


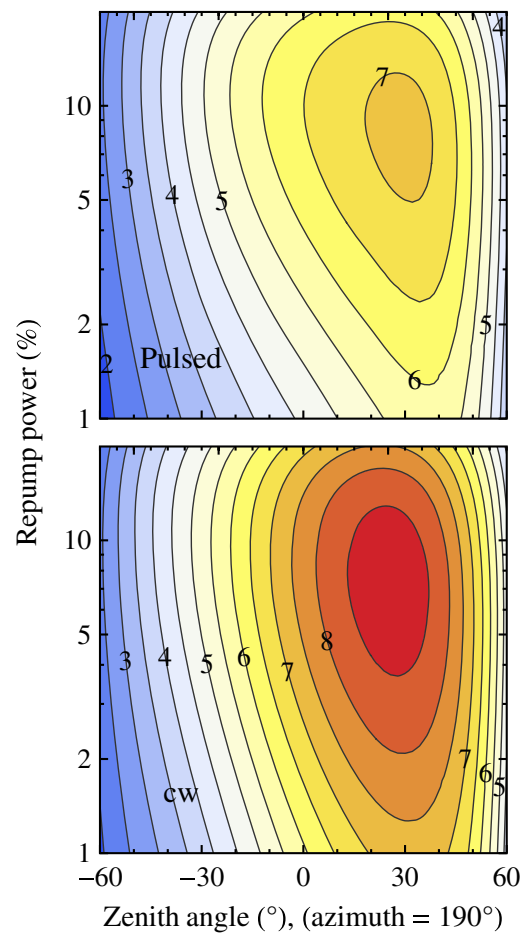

Fig. 19. (Color online) Return flux in units of $10^{6}$ photon $/ \mathrm{s} / \mathrm{m}^{2}$ as a function of zenith angle and repump power fraction for the Mauna Kea site. Upper plot is for pulsed light, and the lower plot is for $\mathrm{cw}$.

we see that increasing the line width of the modes of the pulsed laser beyond the current assumed value of $15 \mathrm{MHz}$ would result in reduced flux return for many observation directions.

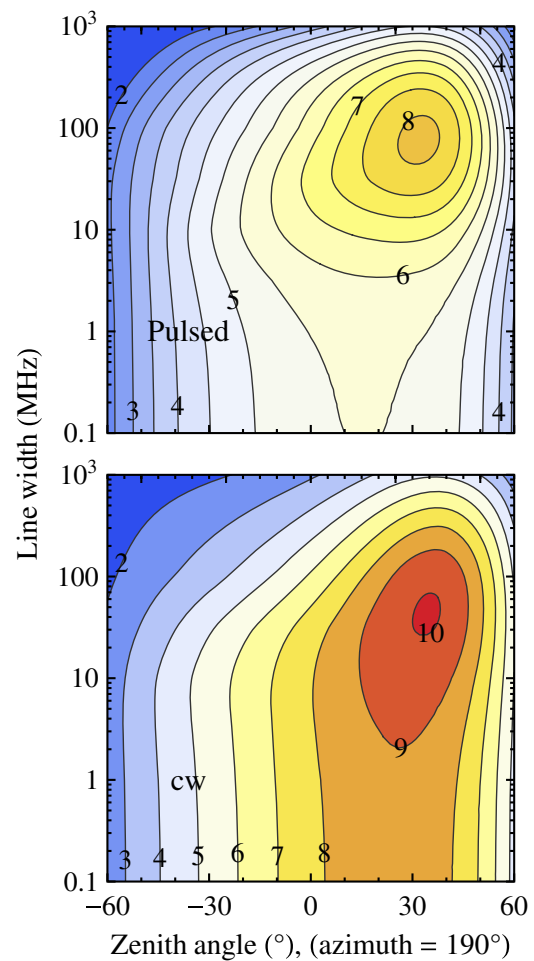

Fig. 20. (Color online) Return flux in units of $10^{6}$ photon $/ \mathrm{s} / \mathrm{m}^{2}$ as a function of zenith angle and line width of each mode for the Mauna Kea site. Upper plot is for pulsed light, and lower plot is for $\mathrm{cw}$. The natural line width of the $\mathrm{Na} \mathrm{D}_{2}$ transition is $\sim 10 \mathrm{MHz}$.

\section{CONCLUSIONS}

Numerical simulations of photon return using both a pulsed laser with specifications provided by TIPC and a narrow-band cw laser have been performed for the Mauna Kea and LZT sites. The results indicate that, if repump light is used, both the cw and pulsed formats may be able to meet the design requirements for photon return. Within the level of uncertainty in the model, the $\mathrm{cw}$ laser format provides comparable return to the pulsed format.

We have also investigated the dependence of the return flux on parameters such as the pulse length, the fraction of laser power dedicated to repumping, and the laser line width. For the case of the laser line width, we found that the optimal value has a strong dependence on the angle between the light propagation direction and the magnetic-field direction.

Future modeling work could use time-resolved measurements of the mode structure of the TIPC laser to obtain more accurate results. Another avenue of research could be to quantify the impact of changing environmental conditions on the photon return, for example, the sodium-layer density profile, optical seeing conditions, and uncertainties in collision rates.

Finally, experimental validation of the model will greatly aid future development. Published reports of comparison between theoretical models and measurements for Na LGS systems have been limited; there are some results for a cw LGS at the Starfire Optical Range [40] and for a transportable cw LGS developed by the European Southern Observatory (ESO) [41]. Significant information can be obtained even in the absence of absolute sodium density measurements: by measuring the dependence of the photon flux on experimentally variable parameters, such as zenith angle, light power, light polarization, and repump power fraction, the accuracy of various aspects of the model may be verified, providing information about the accuracy of the absolute photon return values obtained from the simulation.

\section{ACKNOWLEDGMENTS}

The TMT Project gratefully acknowledges the support of the TMT collaborating institutions. They are the Association of Canadian Universities for Research in Astronomy (ACURA), the California Institute of Technology, the University of California, the National Astronomical Observatory of Japan, the National Astronomical Observatories of China and their consortium partners, and the Department of Science and Technology of India and their supported institutes. This work was supported as well by the Gordon and Betty Moore Foundation, the Canada Foundation for Innovation, the Ontario Ministry of Research and Innovation, the National Research Council of Canada, the Natural Sciences and Engineering Research Council of Canada, the British Columbia Knowledge Development Fund, the Association of Universities for Research in Astronomy (AURA) and the U.S. National Science Foundation. The authors also acknowledge the invaluable assistance of the Technical Institute of Physics and Chemistry of the Chinese Academy of Sciences.

\section{REFERENCES}

1. Astronomy and Astrophysics Survey Committee, Board on Physics and Astronomy, Space Studies Board, National Research 
Council, Astronomy and Astrophysics in the New Millennium (The National Academies Press, 2001).

2. Committee for a Decadal Survey of Astronomy and Astrophysics; National Research Council, New Worlds, New Horizons in Astronomy and Astrophysics (The National Academies Press, 2010).

3. M. Schöck, S. Els, R. Riddle, W. Skidmore, T. Travouillon, R. Blum, E. Bustos, G. Chanan, S. G. Djorgovski, P. Gillett, B. Gregory, J. Nelson, A. Otárola, J. Seguel, J. Vasquez, A. Walker, D. Walker, and L. Wang, "Thirty meter telescope site testing I: overview," Publ. Astron. Soc. Pac. 121, 384-395 (2009).

4. D. T. Gavel, E. L. Gates, C. E. Max, S. S. Olivier, B. J. Bauman, D. M. Pennington, B. A. Macintosh, J. Patience, C. G. Brown, P. M. Danforth, R. L. Hurd, S. A. Severson, and J. P. Lloyd, "Recent science and engineering results with the laser guidestar adaptive optic system at Lick Observatory," Proc. SPIE 4839, 354-359 (2003).

5. M. Le Louarn, N. N. Hubin, and R. Arsenault, "Adaptive optics for second-generation VLT instruments," Proc. SPIE 5490 248-259 (2004).

6. P. L. Wizinowich, D. L. Mignant, A. H. Bouchez, R. D. Campbell, J. C. Y. Chin, A. R. Contos, M. A. v. Dam, S. K. Hartman, E. M. Johansson, R. E. Lafon, H. Lewis, P. J. Stomski, D. M. Summers, C. G. Brown, P. M. Danforth, C. E. Max, and D. M. Pennington, "The W. M. Keck Observatory laser guide star adaptive optics system: oerview," Publ. Astron. Soc. Pac. 118, 297-309 (2006).

7. M. Boccas, F. Rigaut, M. Bec, B. Irarrazaval, E. James, A. Ebbers, C. d'Orgeville, K. Grace, G. Arriagada, S. Karewicz, M. Sheehan, J. White, and S. Chan, "Laser guide star upgrade of Altair at Gemini North," Proc. SPIE 6272, 62723L (2006).

8. M. Hart, N. M. Milton, C. Baranec, K. Powell, T. Stalcup, D. McCarthy, C. Kulesa, and E. Bendek, "A ground-layer adaptive optics system with multiple laser guide stars," Nature $\mathbf{4 6 6}$, 727-729 (2010).

9. M. Lloyd-Hart, R. Angel, N. M. Milton, M. Rademacher, and J. Codona, "Design of the adaptive optics systems for GMT," Proc. SPIE 6272, 62720E (2006).

10. B. Ellerbroek, S. Adkins, D. Andersen, J. Atwood, C. Boyer, P. Byrnes, R. Conan, L. Gilles, G. Herriot, P. Hickson, E. Hileman, D. Joyce, B. Leckie, M. Liang, T. Pfrommer, J.-C. Sinquin, J.-P. Veran, L. Wang, and P. Welle, "Progress toward developing the TMT adaptive optical systems and their components," Proc. SPIE 7015, 70150R (2008).

11. E. Diolaiti, J.-M. Conan, I. Foppiani, M. Lombini, C. Petit, C. Robert, L. Schreiber, P. Ciliegi, E. Marchetti, M. Bellazzini, L. Busoni, S. Esposito, T. Fusco, N. Hubin, F. QuirosPacheco, A. Baruffolo, S. D'Odorico, J. Farinato, B. Neichel, R. Ragazzoni, C. Arcidiacono, V. Biliotti, G. Bregoli, G. Cosentino, and G. Innocenti, "A preliminary overview of the multiconjugate adaptive optics module for the E-ELT," Proc. SPIE 7015, 70150U (2008).

12. L. A. Thompson and R. M. Castle, "Experimental demonstration of a Rayleigh-scattered laser guide star at $351 \mathrm{~nm}$," Opt. Lett. 17, 1485-1487 (1992).

13. T. H. Jeys, "Development of a mesospheric sodium laser beacon for atmospheric adaptive optics," Lincoln Lab. J. 4, 133-150 (1991).

14. W. Happer, G. J. MacDonald, C. E. Max, and F. J. Dyson, "Atmospheric-turbulence compensation by resonant optical backscattering from the sodium layer in the upper atmosphere," J. Opt. Soc. Am. A 11, 263-276 (1994).

15. R. Foy and A. Labeyrie, "Feasibility of adaptive telescope with laser probe," Astron. Astrophys. 152, L29-L31 (1985).

16. R. Q. Fugate, D. L. Fried, G. A. Ameer, B. R. Boeke, S. L. Browne, P. H. Roberts, R. E. Ruane, G. A. Tyler, and L. M. Wopat, "Measurement of atmospheric wavefront distortion using scattered light from a laser guide-star," Nature 353, 144-146 (1991).

17. C. Steidel (2009). (From a talk about the TMT Science Goals before the GSMT Community Assessment Review (GCAR) Panel, Pasadena).

18. K. Matthews, A. M. Ghez, A. J. Weinberger, and G. Neugebauer, "The first diffraction-limited images from the W. M. Keck Telescope," Publ. Astron. Soc. Pac. 108, 615-619 (1996).

19. R. Schödel, T. Ott, R. Genzel, R. Hofmann, M. Lehnert, A. Eckart, N. Mouawad, T. Alexander, M. J. Reid, R. Lenzen, M. Hartung,
F. Lacombe, D. Rouan, E. Gendron, G. Rousset, A.-M. Lagrange, W. Brandner, N. Ageorges, C. Lidman, A. F. M. Moorwood, J. Spyromilio, N. Hubin, and K. M. Menten, "A star in a 15.2-year orbit around the supermassive black hole at the centre of the Milky Way," Nature 419, 694-696 (2002).

20. A. M. Ghez, S. Salim, N. N. Weinberg, J. R. Lu, T. Do, J. K. Dunn, K. Matthews, M. R. Morris, S. Yelda, E. E. Becklin, T. Kremenek, M. Milosavljevic, and J. Naiman, "Measuring distance and properties of the Milky Way's central supermassive black hole with stellar orbits," Astrophys. J. 689, 1044-1062 (2008).

21. L. Gilles, L. Wang, and B. L. Ellerbroek, "Impact of laser launch location on the performance of laser tomography and multiconjugate adaptive optics for extremely large telescopes," Appl. Opt. 49, G114-G119 (2010).

22. L. Wang, A. Otarola, and B. Ellerbroek, "Impact of sodium laser guide star fratricide on multi-conjugate adaptive optics systems," J. Opt. Soc. Am. A 27, A19-A28 (2010).

23. C. Boyer, B. Ellerbroek, L. Gilles, and L. Wang, "The TMT laser guide star facility," in First AO4ELT Conference-Adaptive Optics for Extremely Large Telescopes (EDP Sciences, 2010), p. 04004.

24. D. L. Fried, "Optical resolution through a randomly inhomogeneous medium for very long and very short exposures," J. Opt. Soc. Am. 56, 1372-1379 (1966)

25. L. Gilles and B. Ellerbroek, "Shack-Hartmann wavefront sensing with elongated sodium laser beacons: centroiding versus matched filtering," Appl. Opt. 45, 6568-6576 (2006).

26. R. Holzlöhner, S. M. Rochester, D. Bonaccini Calia, D. Budker, J. M. Higbie, and W. Hackenberg, "Optimization of cw sodium laser guide star efficiency," Astron. Astrophys. 510, A20 (2010).

27. K. Avicola, J. M. Brase, J. R. Morris, H. D. Bissinger, J. M. Duff, H. W Friedman, D. T. Gavel, C. E. Max, S. S. Olivier, R. W. Presta, D. A Rapp, J. T. Salmon, and K. E. Waltjen, "Sodium-layer laser-guidestar experimental results," J. Opt. Soc. Am. A 11, 825-831 (1994)

28. P. W. Milonni, R. Q. Fugate, and J. M. Telle, "Analysis of measured photon returns from sodium beacons," J. Opt. Soc. Am. A 15, 217-233 (1998).

29. J.-P. Pique and S. Farinotti, "Efficient modeless laser for a mesospheric sodium laser guide star," J. Opt. Soc. Am. B 20, 2093-2101 (2003).

30. E. Kibblewhite, "The physics of the sodium laser guide star: predicting and enhancing the photon returns of sodium guide stars for different laser technologies," in Proceedings of the Advanced Maui Optical and Space Surveillance Technologies Conference, S. Ryan, ed. (Curran Associates, 2009), p. E33.

31. W. Happer, Y.-Y. Jau, and T. Walker, Optically Pumped Atoms (Wiley-VCH, 2010).

32. A. Corney, Atomic and Laser Spectroscopy (Clarendon, 2006).

33. Available at http://budker.berkeley.edu/ADM/.

34. Available at http://computation.llnl.gov/casc/sundials/.

35. A. C. Hindmarsh, P. N. Brown, K. E. Grant, S. L. Lee, R. Serban, D. E. Shumaker, and C. S. Woodward, "SUNDIALS: suite of nonlinear and differential/algebraic equation solvers," ACM Trans. Math. Softw. 31, 363-396 (2005).

36. Available at http://omniweb.gsfc.nasa.gov/vitmo/msis \_vitmo .html.

37. V. I. Tatarski, Wave Propagation in a Turbulent Medium Translated from Russian by R. A. Silverman (McGraw-Hill, 1961).

38. R. Holzlöhner, S. M. Rochester, T. Pfrommer, D. B. Calia D. Budker, J. M. Higbie, and W. Hackenberg, "Laser guide star return flux simulations based on observed sodium density profiles," Proc. SPIE 7736, 77360V (2010).

39. This formula for airmass dependence neglects the curvature of the Earth, which is a reasonable assumption for the range of zenith angles $\left(\leq 60^{\circ}\right)$ considered in this study.

40. P. D. Hillman, J. D. Drummond, C. A. Denman, and R. Q. Fugate, "Simple model, including recoil, for the brightness of sodium guide stars created from cw single frequency fasors and comparison to measurements," Proc. SPIE 7015, 70150L (2008).

41. D. B. Calia, R. Holzlöhner, I. Guidolin, F. Pedichini, M. Centrone T. Kasper, G. Lombardi, W. Forth, S. Lewis, T. Pfrommer, and W. Hackenberg, "ELT LGS-AO: optimizing the LGS return flux," in Second International Conference on Adaptive Optics for Extremely Large Telescopes (25-30 Sept. 2011, Victoria, BC, Canada) (to appear). 